\title{
ANALYSIS OF PROTEINS AND PEPTIDES BY MASS SPECTROMETRY
}

\author{
Marianna Trebuňová \\ Technical University of Košice, Faculty of Mechanical Engineering, Department of Biomedical Engineering and \\ Measurement, Letná 9, 04200 Košice, Slovakia, marianna.trebunova @ tuke.sk (corresponding author)

\section{Jozef Živčák} \\ Technical University of Košice, Faculty of Mechanical Engineering, Department of Biomedical Engineering and \\ Measurement, Letná 9, 04200 Košice, Slovakia, jozef.zivcak@tuke.sk
}

Keywords: proteins, peptides, mass spectrometry

Abstract: Analysis of proteins and peptides by mass spectrometry became possible by the end of the 20th century. MS z is a method of analytical chemistry. It works with a $m / Q$ ratio where $m$ is the mass and $Q$ is the fragment charge. It is used to determine the mass of particles, to determine the elemental composition of a sample or molecule, and to elucidate the chemical structure of molecules such as peptides and other chemical compounds. Its principle is based on ionizing chemical compounds and measurements of their mass relative to charge.

\section{Introduction, analysis and identification}

Analysis of proteins and peptides by mass spectrometry became possible by the end of the $20^{\text {th }}$ century. MS $\mathrm{z}$ is a method of analytical chemistry. It works with a $m / Q$ ratio where $m$ is the mass and $Q$ is the fragment charge. It is used to determine the mass of particles, to determine the elemental composition of a sample or molecule, and to elucidate the chemical structure of molecules such as peptides and other chemical compounds. Its principle is based on ionizing chemical compounds and measurements of their mass relative to charge.

1. The sample is placed in the apparatus and undergoesevaporation.

2. The sample components are ionized in one of a number of ways (for example, the impact of an electron beam), resulting in the formation of charged particles - ions.

3. The ions are separated according to the $m / Q$ ratio in the electromagnetic field analyzer.

4. Ions are detected, usually by a quantitative method.

5. Ion is processed by mass spectrometer, Figure 1 [1].

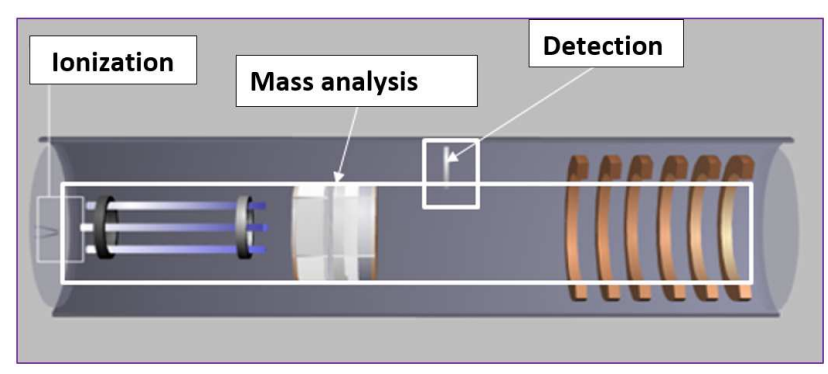

Figure 1 Basic parts of MS [2]

The mass spectrometer consists of three modules:

- The first module is a source of ions that can convert gas molecules to ions

- The second module is a mass analyzer that sorts the ions by their mass using electromagnetic fields
- The third module is a detector that measures the amount of the indicator quantity and thus provides the data to calculate the abundance of each ion in real time.

Technique has qualitative and quantitative use. These include identifying unknown substances, determining the isotopic composition of the elements in the molecule, and determining the structure of the compound by observing its fragmentation. Further use involves detecting the quantitative amount of the mixture in the samples, or studying the gas phase ion bases (ion and neutral molecules in vacuum). Mass spectrometry is commonly used in analytical laboratories that study the physical, chemical or biological properties of various compounds.

\subsection{Steps of mass spectrometry}

In the Figure 2 you can see the graphical representation of individual steps of mass spectrometry.

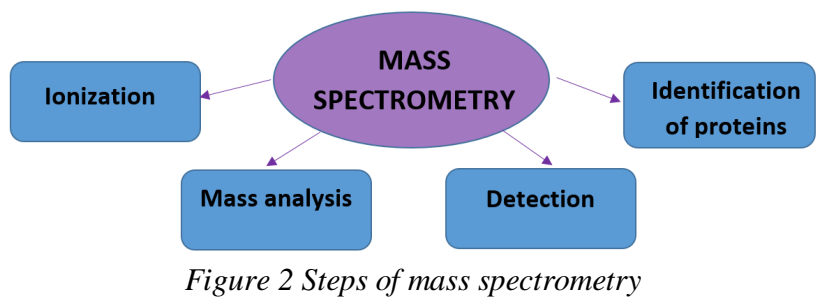

\subsubsection{Ionization}

The ion source is part of the mass spectrometer. This component ionizes the material by analysis (analyte). The ions are then transported by magnetic or electric fields to a mass analyzer. Ionization techniques have been the key to determining what types of samples can be analyzed by mass spectrometry. Electrone and chemical ionization are used for gases and vapors. In the chemical ionization of sources, the analyte is ionized by chemical ion-reacting 
ANALYSIS OF PROTEINS AND PEPTIDES BY MASS SPECTROMETRY

Marianna Trebuňová; Jozef Živčák

molecules during collisions in the source. Examples of ion sources are:

\section{ESI ("electrospray ionization")}

For ESI, a sample of proteins dissolved in a solvent (liquid medium), in a volume of about $1 \mu \mathrm{l}$, is placed in the capillary. The conically tapered capillary allows a sample flow rate of 20-40 $\mathrm{nl} / \mathrm{min}$, the volume of the sample can be analyzed for up to 1 hour. The liquid is drawn into the electrostatic field, the droplets are sucked in by the drying air, the solvent evaporates and the particle breaks up, increasing the repulsive forces. Repeating this process makes it possible to obtain the ions of proteins (charged molecules) that move in the vacuum tube, the process zones and their movement is directed by the electric field. Analyzed molecules can carry multiple charges. This type of ionization and mass analyzers allows the analysis of proteins with molecular weight to $5000[3,4]$.

\section{MALDI (matrix-assisted laser desorption/ionization).}

For MALDI, the sample is dissolved in a volume of about 1-2 $\mu 1$, mixed with the same volume of a matrix solution containing an organic substance (e.g., 3,5dimethoxy-4-OH-cinnamic or 2,5-dihydroxybenzoic acid) about a solid environment. The sample solution is applied to a target and dried at room temperature. The sample crystals are broken down by a small-laser laser and released from the target. The ions are moved in the vacuum tube and the movement is directed by the electric field. The time at which the ions reach the detector is proportional to the second power of the mass / charge ratio $(\mathrm{m} / Q)$. Smaller proteins, therefore, make the detector much longer (time of flight) on the basis of which we can determine their mass. This type of MS allows the analysis of proteins with a molecular weight of up to 100,000 , Figure 3 [3].

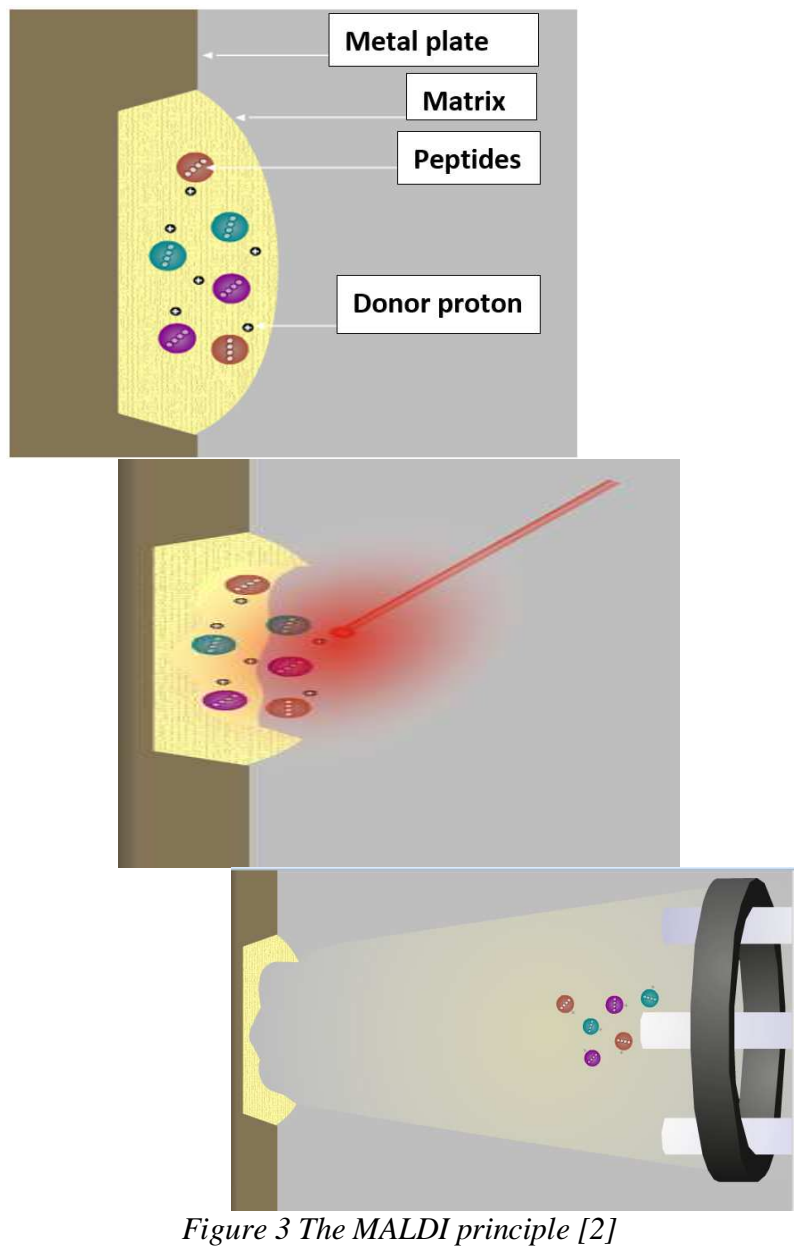

\subsubsection{Mass analysis}

Mass analyzers separate the ions according to their $m / Q$ ratio. The following two laws (1), (2) are governed by the dynamics of charged particles in electric and magnetic fields in vacuum:

$$
F=Q(E+v \times B)
$$

Lorentz's law of power

$$
F=m \cdot a \quad[N]
$$


ANALYSIS OF PROTEINS AND PEPTIDES BY MASS SPECTROMETRY

Marianna Trebuňová; Jozef Živčák

Newton's second movement law in an unrealistic case, i. only applies at an ion rate significantly lower than the speed of light

$F$ - ionic force,

$m$ - the mass of ions,

$a$ - acceleration,

$Q$ - ion charge,

$E$ - electric field,

$v \times B$ - vector product of velocity ions and magnetic field.

The sign of equality between the expressions for the above forces acting on the ion implies (3):

$$
(m / Q) \cdot a=E+v \times B
$$

This differential equation is the classical motion equation for charged particles. Together with the particles of initial conditions, the particles completely determine the motion in space and time as regards the $m / Q$ ratio [5].

There are many types of mass analyzers: static or dynamic, magnetic or electrical, but all operate according to the above differential equation. Each type of analyzer has its strengths and weaknesses. Many mass spectrometers use two or more mass analyzers for tandem mass spectrometry (MS / MS). There are also other types of analyzers eg:

\section{Quadrupole}

The four-pole mass analyzer uses an oscillating electric field to selectively stabilize or destabilize the ion path by radio frequency, the electric field being formed from four parallel electrode rods, alternating at different frequencies. Only ions within a certain range of $m / Q$ ratios are sold through the system at any time. Potential changes on the electrodes allow a wide range of $m / Q$ values. The four-pole filter acts as a selective filter in the mass analyzer and is closely associated with the quad-core ion trap (Figure 4). The other variations of the four-pole are threequadruples.

Triple quadrupole mass spectrometers have three consecutive quadruples arranged in series. The first quadrupole acts as a mass filter, the second acts as a collision target, where the ions are selected into fragments. The resulting fragments are analyzed by the third quadrupole [5].

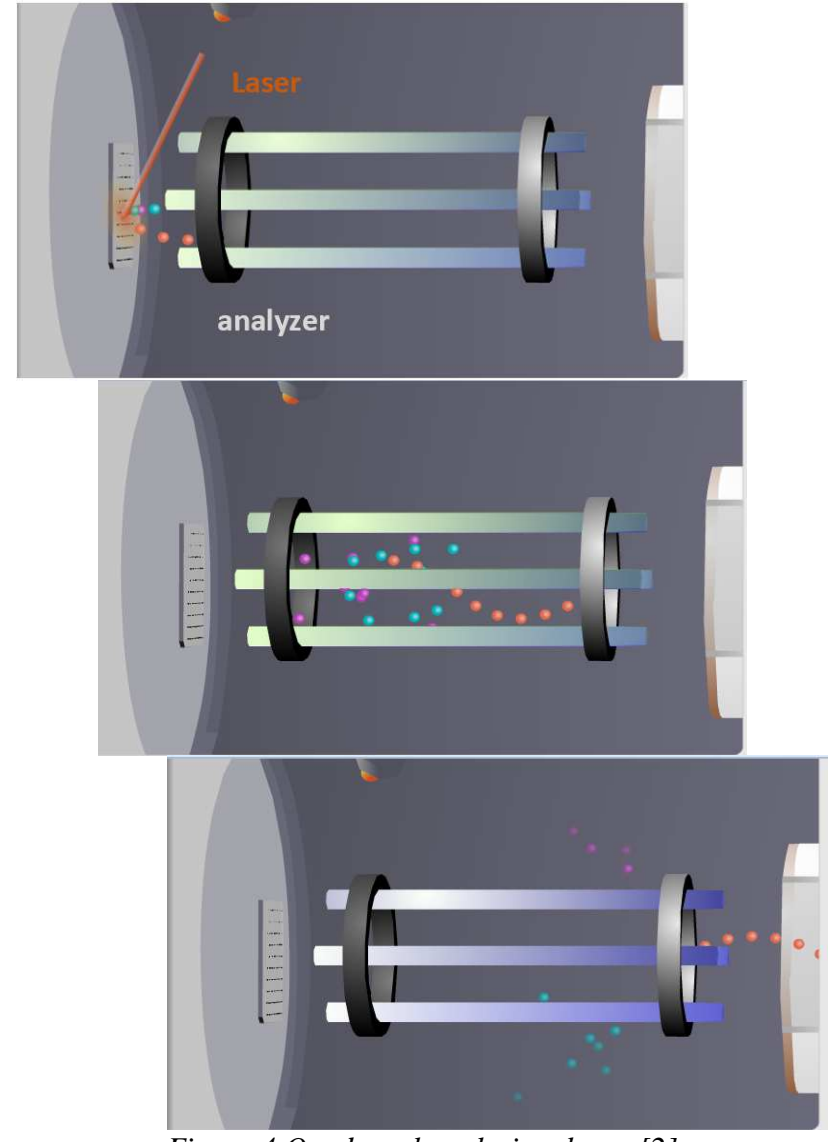

Figure 4 Quadrupol analysis scheme [2]

\section{Quadrupole Ion Trop}

The four-pole ionic trap operates with the same physical laws as four-pole mass analyzers, but the ions are trapped and subsequently evaluated. The sample is ionized either internally (e.g., electron or laser beam) or externally, in which case the ions are often introduced through the hole to the so-called "face" of the electrode.

There are many weight separation and isolation methods, but the most unstable is a mass unstable mode in which the radio frequency potential is maintained such that the orbits of the mass "a" " $b$ " are stable while the ions with the mass "b" < "a" are unstable and are catapulted to the z-axis on the detector. Non-destructive methods of analysis are also used there.

The ions can also be evaluated by the resonant excitation method when the additional oscillation excitation voltage is applied to the face of the electrode, and the amplitude capture and / or frequency of the excitation voltage are manifoldly transferred to the resonant state in order of their $m / Q$ ratio. The linear fourpole ionic trap is similar to a four-pole ion trap, but it captures the ions in a two-dimensional four-pole array instead of three-dimensional quadruple fields as in a 3D four-pole ion trap. An example of a linear ion trap is Thermo Fisher LTQ [5]. 
Time of Flight Mass Spectrometry (TOF-MS)

TOF - MS uses the differences in times when the ions pass through the separation field, and they are separated by mass. It works in pulse mode, so the ions have to be formed or must be extracted from the ion source in pulse mode. The electric field accelerates all ions to the unbounded area of drifting with kinetic energy $z . V(4)$ where $z$ is ion charge and $V$ is the applied voltage. Since the kinetic energy of the ion is $\frac{1}{2} m v^{2}$, the more easier the ions have a greater velocity than the heavier ions, and the detector reaches the end of the drift area earlier.

$$
E_{\text {kin }}=\frac{1}{2} m v^{2}=z \cdot V \quad[J]
$$

The speed of the moving ions is proportional to the second power of the ratio of their double kinetic energy and the mass of the ion (5):

$$
v=\sqrt{\frac{2 \cdot z \cdot V}{m}} \quad\left[m s^{-1}\right] \quad t=\frac{L}{\sqrt{\frac{2 V}{m / z}}}
$$

The flight time $t$ through the drift tube is $L / V$, where $L$ is the length of the drifting tube.

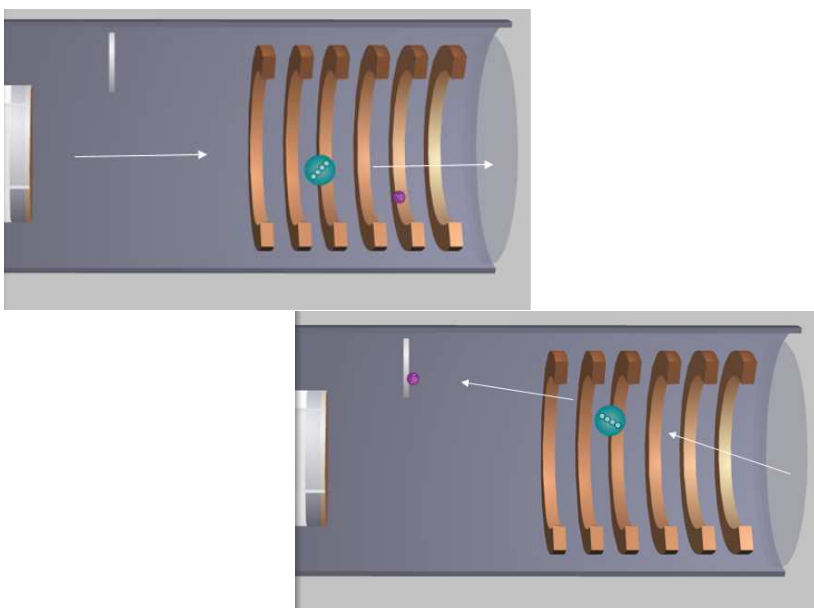

Figure 5 The scheme of TOF analyzer for mass, before and after protein discoloration [7]

The flight time analyzer uses an electric field to accelerate ions with the same potential. If the particles have the same charge, the kinetic energy of the particles will be identical, and their velocity will depend only on their weight. Easier ions reach the first detector (Figure 5) [5].

\subsubsection{Detection}

The last element of the mass spectrometer is the detector. Recognizes induced or current ion passes or their effects. The detector consists of two metal plates that record the passage of ions. The ion current on the detection slot is very small (approximately $10^{-9}-10^{-18} \mathrm{~A}$ ). Therefore, a photoelectronic multiplier is used which provides an increase of up to about $10^{-6}$.
As a record of mass spectra, fast mirror galvanometers were used in the past to record on photographic paper that was developing itself in the light. Currently PC computing technique is used and spectra are obtained both in tabular form and graphically in standard form $[5,6]$.

\subsubsection{Protein identification}

In the Figure 6 you can see the diagram of proteins identification.

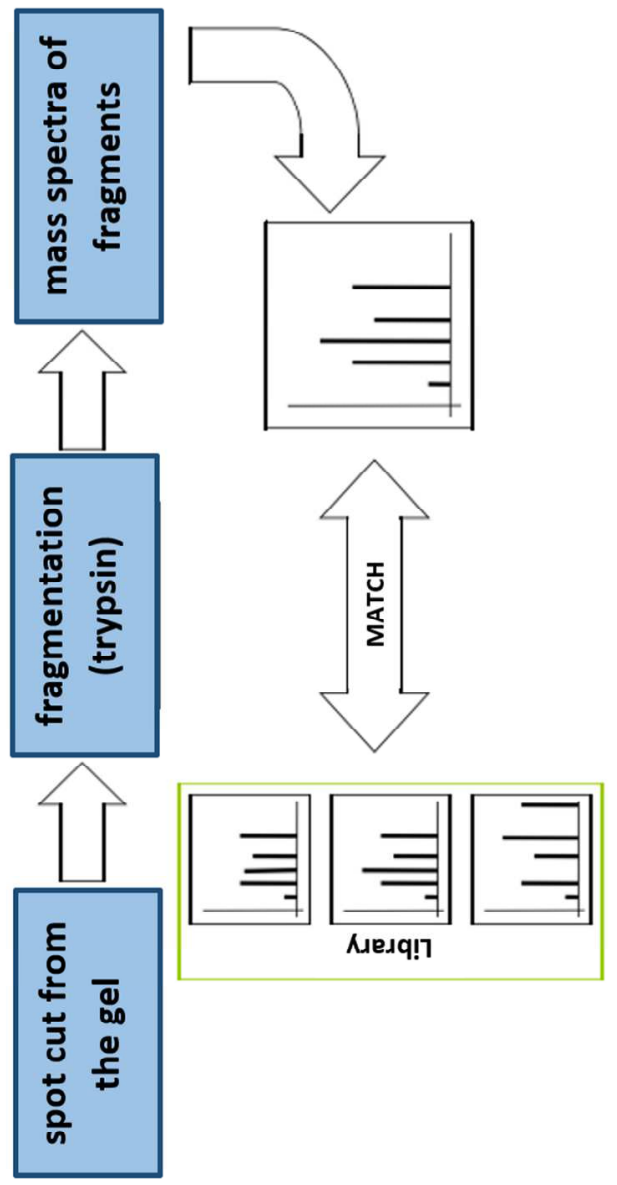

Figure 6 Diagram of proteins identification [1]

There are several ways that MS use to identify proteins. You are here for example:

Peptide mass fingerprinting Maldi Time of Ftight (peptides generated by digestion can be determined by mass spectrometry using peptide mass spectra) utilizes the masses of proteolytic peptides as input for searching in the database of predicted values generated by the digestion of known proteins. If the protein sequence is in the database list, there is evidence that this protein is present in the original sample. 


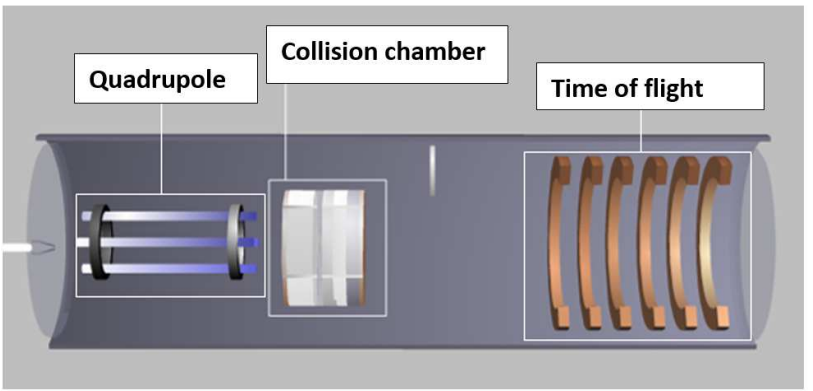

Figure 7 The scheme of Tandem Mass Spectrometry Quadrupole Time of Flight [7]

Tandem Mass Spectrometry is an increasingly popular experimental method used to identify proteins, characterize their amino acid sequences, and posttranslational modifications. Collision-induced dissociation is used in conventional applications to generate a fragment of a particular peptide ion. The fragmentation process results in the cleavage of products by electron capture which decomposes along the peptide bond in the gas phase. With such unnecessary fragmentation, it is possible to use the observed fragments from the mass to match the predicted sequence database to one of the many peptide sequences listed. This technique is referred to as a "topdown or botton-up" approach, because starting with the whole mass and then pulling it from one another, proteolytic fragments are cracked, the protein being assembled together by de novo repeated detection, Figure 7 $[3,7]$.

\section{Conclusions}

Today, we are aware that the path from genomics to proteomics would not have been possible without the application of improved special techniques using mass spectrometry principles. There is probably no other current technique that would overcome the mass spectrometer in a variety of applications in basic and applied research as well as in diagnostics.

\section{Acknowledgement}

This article was supported by the state grants KEGA 069TUKE-4/2017(50\%) of the Ministry of Education of the Slovak Republic and APVV-15-0356 (50\%) MEDPEEK.

\section{References}

[1] http://sk.wikipedia.org/wiki/Hmotnostn\%C3\%A1_spe ktrometria, 2018. (Original in Slovak)

[2] http://proteomics.cancer.gov/whatisproteomics/videot utorial, 2018.

[3] www.fnspba.sk/aktuality-subory/postery/C19.ppt, 2018. (Original in Slovak)

[4] http://www.biocenter.sk/jnteaching_files/Proteomika_ 2013_handouts.pdf, 2018.

[5] http://sk.wikipedia.org/wiki/Hmotnostn\%C3\%A1_spe ktrometria, 2018. (Original in Slovak)

[6] KURUC, J: Úvod do hmotnostnej spektrometrie, pp. 118,2004 . (Original in Slovak)

[7] http://www.chromatographyonline.com/lcge/Misc/Top -Down-versus-Bottom-Up-Approaches-inroteomics/ArticleStandard/Article/detail/374600, 2014.

\section{Review process}

Single-blind peer review process. 